\title{
Multipole Theory and Algorithms for Target Support Estimation
}

\author{
Edwin A. Marengo \\ Department of Electrical and Computer Engineering, Northeastern University, Boston, MA 02115, USA \\ Correspondence should be addressed to Edwin A. Marengo; emarengo@ece.neu.edu
}

Received 25 April 2013; Accepted 1 July 2013

Academic Editor: Francesco Soldovieri

Copyright (C) 2013 Edwin A. Marengo. This is an open access article distributed under the Creative Commons Attribution License, which permits unrestricted use, distribution, and reproduction in any medium, provided the original work is properly cited.

\begin{abstract}
The inverse problem of estimating the smallest region of localization (minimum source region) of a source or scatterer that can produce a given radiation or scattered field is investigated with the help of the multipole expansion. The results are derived in the framework of the scalar Helmholtz equation. The proposed approach allows the estimation of possibly nonconvex minimum source regions. The derived method is illustrated with an example relevant to inverse scattering.
\end{abstract}

\section{Introduction}

It has been shown (see $[1,2])$ that, given the far field associated to an unknown radiating source, it is possible to uniquely define the minimum convex source region associated to the given far field. The theoretical principles can be traced back to the treatise of Müller ([3, ths. 26, 27, 29]) who used the spherical wave or multipole expansion of the radiated field to show that, for a fixed origin of coordinates, say $O$, there is a minimum spherical source region of radius $R_{\min }, B_{\min }(O)=$ $\left\{\mathbf{r} \in \mathbb{R}^{3}: r \equiv|\mathbf{r}| \leq R_{\min }(O)\right\}$, such that, in order for a source to be able to produce the given far field radiation pattern, it must lie in the interior of a spherical volume centered about the same origin and having a radius that is at least as large as $R_{\min }$. Furthermore, the radiated field outside $B_{\min }$ is an analytic function that can be analytically continued up to the boundary of a minimum source region $V_{\text {min }}$ located inside $B_{\min }$; see [4, page 143]. Figure 1(a) illustrates the minimum source region and the respective minimum spherical source region corresponding to a given origin. From the point of view of propagation or diffraction, then the far field can be inverse-diffracted up to the boundary of $V_{\min } \subseteq B_{\min }$. In addition, clearly if one considers another origin $O^{\prime}$, then one can also find another minimum spherical volume $B_{\min }\left(O^{\prime}\right)$ centered about this origin and so on. Then if one considers a set of, say, $n$ origins and $n$ associated minimum spherical volumes, the intersection of all such source regions $\mathscr{B}_{\text {min }}$ establishes a sharper bound on the minimum source region $V_{\text {min }} \subseteq \mathscr{B}_{\text {min }}$ which lies in the interior of such intersection, as is illustrated in Figure 1(b). By means of this procedure it is theoretically possible to uniquely define and compute the convex scattering support or minimum convex source region, say $B_{\text {min,conv }} \supseteq V_{\min }$, which is a subset of the convex hull of any source producing the given far field. This minimum convex source region is the smallest convex support of any source producing that field and represents important source localization information that can be inverted uniquely from the far field data despite the general nonuniqueness (without further priors) of the full inverse source problem of reconstructing the actual source that generated the given far field; see, for example, $[5,6]$ for discussions of the inverse source problem and the relevant nonradiating sources corresponding to the null space of the forward source-to-field mapping. In this connection, it is worth commenting that for zero far fields the minimum source region does not exist (empty set). Any nonradiating source can produce that trivial far field, and of course no source at all also produces that same far field. However, for nontrivial far fields (associated to fields obeying the radiation condition) the minimum source region is a nonempty set (since, as is well known, the only solution to the homogeneous Helmholtz equation obeying the radiation condition is the zero field). Consequently, here and henceforth we implicitly assume nontrivial far fields, for which one can always define corresponding minimum source regions.

The method in [1] applies to the minimum convex source region. For geometrical reasons, this plane wave expansion approach cannot be used to estimate nonconvex minimum 


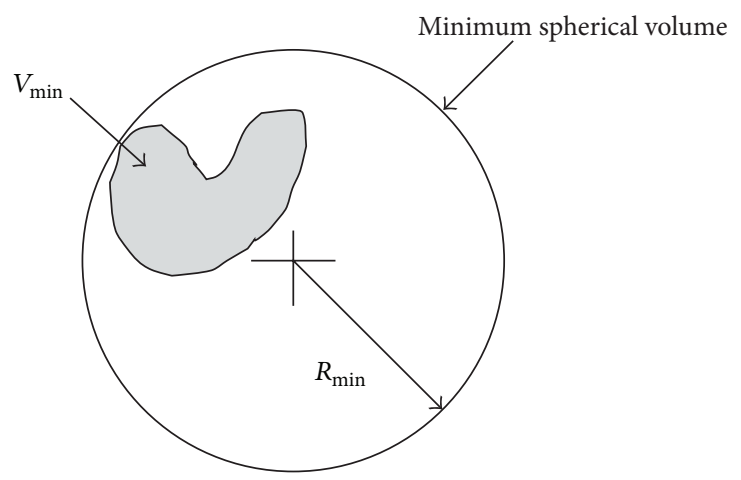

(a) Minimum source region

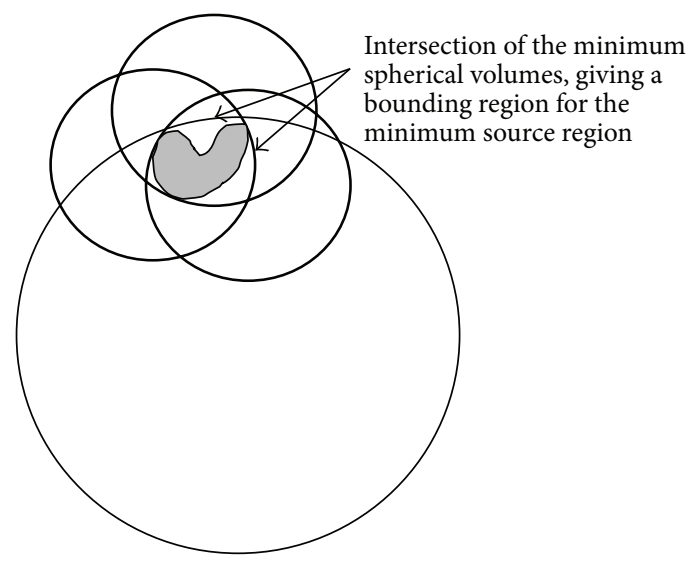

(b) Bound using multiple origins

Figure 1: (a) Minimum source region $V_{\min }$ and the minimum spherical volume $B_{\min }(O)$ for a given origin $O$. (b) Bounding region for the minimum source region obtained from the intersection of minimum spherical source regions for different origins.

source regions. In this paper we use the multipole expansion to obtain the respective closed form formula to estimate the full minimum source region including nonconvex minimum source regions that cannot be derived with the existing methods. To achieve this goal, first we derive the respective multipole theory counterpart of the key formula (equation (6)) in [1] for estimation of the convex support. Later we derive the respective generalization to tackle the pending nonconvex support, which completes the computation of the minimum source region.

One of the main appeals of the multipole expansion is its validity for both near and far fields; hence the results of this work apply to imaging and inverse scattering with either near or far fields. For example, in the subsurface sensing problem illustrated in Figure 2, probing sources and scattering sensors can be used that are in the near or far zone of the target. The theory and algorithms of this work provide a framework to quantitatively characterize source regions in the investigation domain that must reside in the interior of the scatterer, thereby offering valuable localization information for the extraction of subsequent target information. Among other questions, the role of the probing source positions in rendering target information can be quantitatively investigated with the proposed analytical and numerical tools based on the multipole expansion. This contribution thus

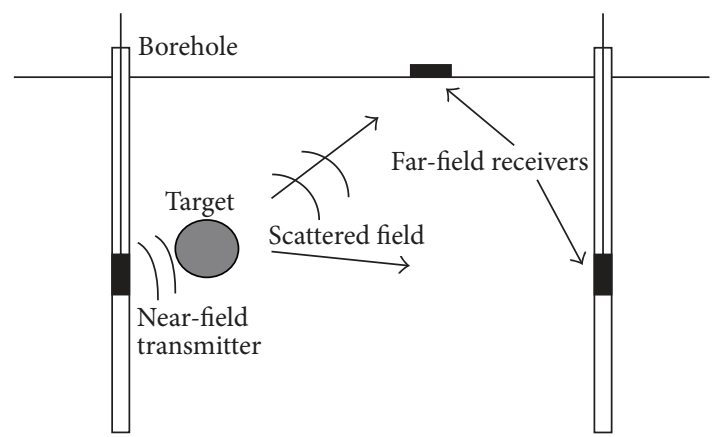

FIGURE 2: Subsurface sensing scenario involving near probing fields and far zone sensors. The multipole theory imaging approach of this paper is useful for imaging with either near or far fields.

sheds light on the information about the support of a source or scatterer that is contained in near or far exterior field data. In particular, even though the inverse source problem exhibits general nonuniqueness, the minimum source region is a piece of information about the sought-after source that can be deduced uniquely from the exterior field. Also, in relation to alternative imaging methods, this approach has the particular appeal that it applies in theory to the extraction of 
information about the support of a scatterer using a single probing field.

The rest of the paper is organized as follows. Section 2 presents the multipole-theory-based approach for estimating bounds for the minimum source region, in theory up to the minimum convex source region. Our main result of this section is given in connection with (17). The physical framework is one of radiation, involving the linear map, from equivalent volume sources confined within given spherical volumes, to the far fields. Section 3 provides an alternative approach based on the complementary point of view of (exterior) propagation or diffraction, involving a map from boundary values of the fields (or equivalent Huygens' surface sources) to the far fields. The main result of this section, given in connection with (44), is equivalent to the previous one in (17). Section 4 provides a complementary, interior diffraction approach which allows the estimation of the possibly nonconvex minimum source region. This approach leads to the key nonconvex support estimation formula (69). The theoretical results in Sections 2-4 emphasize the context of the scalar Helmholtz operator in 3D space. To expand the scope of practical applications and examples that can be handled via the same general theoretical framework, in Section 5 we derive the 2D counterpart of the main results in Sections 2-4 applicable to the 2D Helmholtz operator which is relevant to electromagnetic problems involving transverse magnetic polarization. In Section 6 we discuss the practical computational implementation with an inverse scattering example. Section 7 provides concluding remarks.

\section{Radiation-Based Analysis}

2.1. Multipole Radiation. Consider wavefields $\psi$ described by the scalar Helmholtz equation:

$$
\left(\nabla^{2}+k^{2}\right) \psi(\mathbf{r})=-\rho(\mathbf{r}), \quad \mathbf{r} \in \mathbb{R}^{3},
$$

where $k>0$ is the wavenumber of the field and $\rho$ is a source of support $V$. This source can be an active source (emitter) or an induced source such as a scatterer excited by incident fields. The radiated field $\psi$ obeys Sommerfeld's radiation condition.

Consider the smallest set $B_{R} \equiv\left\{\mathbf{r} \in \mathbb{R}^{3}: r \equiv|\mathbf{r}| \leq R\right\}$ which contains the source support such that $V \subseteq B_{R}$. It is well known ([7, equations (9.93), (9.98)]; [8, equations (3.12), (3.14)]) that the radiated field admits the following multipole expansion for $r>R$ :

$$
\psi(\mathbf{r})=i k \sum_{l=0}^{\infty} \sum_{m=-l}^{l} a_{l, m} h_{l}^{(1)}(k r) Y_{l, m}(\widehat{\mathbf{r}}), \quad r>R,
$$

where $\widehat{\mathbf{r}} \equiv \mathbf{r} / r, h_{l}^{(1)}(\cdot)$ is the spherical Hankel function of the first kind, order $l, Y_{l, m}$ is the spherical harmonic of degree $l$ and order $m$ (see [9, Section 12.6]), and the multipole moments $a_{l, m}$, where $l=0,1, \ldots, \infty ; m=-l,-l+1, \ldots, l-1, l$, are given in terms of the source by

$$
a_{l, m}=\int d r r^{2} j_{l}(k r) \int_{S^{2}} d \widehat{\mathbf{r}} Y_{l, m}^{*}(\widehat{\mathbf{r}}) \rho(\mathbf{r}),
$$

where $j_{l}(\cdot)$ is the spherical Bessel function of the first kind and order $l$ and $S^{2}$ denotes the unit sphere. It follows from the large argument approximation for the spherical Hankel function,

$$
h_{l}^{(1)}(k r) \sim \frac{\exp [i(k r-\pi l / 2)]}{i k r},
$$

that in the far zone the generated field behaves like

$$
\psi(r \widehat{\mathbf{r}}) \sim f(\widehat{\mathbf{r}}) \frac{\exp (i k r)}{r},
$$

where the far field radiation pattern $f(\widehat{\mathbf{r}})$ is given by ([8, equation (3.19)])

$$
f(\widehat{\mathbf{r}})=\sum_{l=0}^{\infty} \sum_{m=-l}^{l}(-i)^{l} a_{l, m} Y_{l, m}(\widehat{\mathbf{r}})
$$

which in view of the orthonormality of the spherical harmonics implies

$$
a_{l, m}=i^{l} \int_{S^{2}} d \widehat{\mathbf{r}} Y_{l, m}^{*}(\widehat{\mathbf{r}}) f(\widehat{\mathbf{r}}) .
$$

The radiated power is proportional to the squared $L^{2}$ norm of the far field radiation pattern, given from (6) and the orthonormality of the spherical harmonics by

$$
\|f\|_{2}^{2}=\int_{S^{2}} d \widehat{\mathbf{r}}|f(\widehat{\mathbf{r}})|^{2}=\sum_{l=0}^{\infty} \sum_{m=-l}^{l}\left|a_{l, m}\right|^{2}<\infty .
$$

It is henceforth assumed that (8) is obeyed.

2.2. Equivalent Minimum Energy Sources. Our radiationbased treatment of the inverse support problem will be based on equivalent sources $\hat{\rho}_{a}$ of support $B_{a} \equiv\left\{\mathbf{r} \in \mathbb{R}^{3}: r \leq a\right\}$ that produce the same far field as $\rho$. They are given by [5]

$$
\hat{\rho}_{a}(\mathbf{r})=M_{B_{a}}(\mathbf{r}) \sum_{l=0}^{\infty} \sum_{m=-l}^{l}\left[\frac{a_{l, m}}{\sigma_{l}^{2}(a)}\right] j_{l}(k r) Y_{l, m}(\widehat{\mathbf{r}}),
$$

where the indicator or masking function $M_{\tau}(\mathbf{r})$ is equal to 1 if $\mathbf{r} \in \tau$ and 0 otherwise and where the singular values $\sigma_{l}(a)$ of the linear source-to-far field mapping are defined by

$$
\sigma_{l}^{2}(a)=\int_{r \leq a} d r r^{2} j_{l}^{2}(k r)=\frac{a^{3}}{2}\left[j_{l}^{2}(k a)-j_{l-1}(k a) j_{l+1}(k a)\right] .
$$

The series expansion (9) defines the so-called minimum $L^{2}$ norm or minimum functional energy source of support $B_{a}$ that produces the given far field. For this representation to be physically meaningful it must be well-behaved as we outline next. 71])

It is easy to show from the asymptotic form (see [3, page

$$
j_{l}(z) \sim \sqrt{\frac{\pi}{2 z}} \frac{(z / 2)^{l+1 / 2}}{\Gamma(l+3 / 2)}, \quad l \longrightarrow \infty,
$$


which implies

$$
\sigma_{l}^{2}(a) \sim \frac{\pi a^{3}(k a)^{2 l}}{2^{2 l+2}(2 l+3) \Gamma^{2}(l+3 / 2)}, \quad l \longrightarrow \infty
$$

plus the property (see [3, Lemma 13])

$$
\left|Y_{l, m}(\widehat{\mathbf{r}})\right| \leq \sqrt{\frac{2 l+1}{4 \pi}}
$$

and standard convergence tests, that, for $a>R$, the series expansion (9) converges absolutely and uniformly in $B_{a} \supset B_{R}$ and the $L^{2}$ norm or functional energy of the equivalent source $\hat{\rho}_{a}$ is finite, that is,

$$
\sum_{l=0}^{\infty} \sum_{m=-l}^{l} \frac{\left|a_{l, m}\right|^{2}}{\sigma_{l}^{2}(a)}<\infty
$$

Thus there is a well-behaved equivalent source $\widehat{\rho}_{a} \in L^{2}\left(B_{a}\right)$ $B_{R}$ ) of the form (9) valid for any original $V$-supported source $\rho$. The two sources $\rho$ and $\widehat{\rho}_{a}$ are equivalent in the sense that they produce the same multipole moments and thereby also the same (exterior) field in $\mathbb{R}^{3} \backslash B_{a}$ :

$$
\psi(r \widehat{\mathbf{r}})=i k \sum_{l=0}^{\infty} \sum_{m=-l}^{l} a_{l, m} h_{l}^{(1)}(k r) Y_{l, m}(\widehat{\mathbf{r}}), \quad r>a .
$$

Furthermore, depending on the particular far field, it is possible that the equivalent source $\widehat{\rho}_{a}$ in (9) remains meaningful, for example, in the finite $L^{2}$ norm sense (14), for some radius $a \leq R$. The infimum of the set of such radii defines the radius $R_{\min }$ of the minimum spherical source region as we elaborate next.

2.3. Minimum Spherical Source Regions. The minimum spherical source region $B_{\text {min }} \equiv\left\{\mathbf{r} \in \mathbb{R}^{3}: r \leq R_{\text {min }}\right\}$ can be estimated by asking the question whether a physically reasonable source of support $B_{\min }$ can be manufactured which produces the desired far field or equivalently the associated multipole moments of the field. We have explored different criteria for the physical reasonableness, concluding that a number of plausible alternatives are equivalent to the canonical Picard's condition defining the range of the linear mapping from $L^{2}$ sources confined within a spherical volume to $L^{2}$ far fields which obey (8). In particular, we require the equivalent source to obey (14). Thus we define the radius $R_{\min }$ of the minimum spherical volume $B_{\min }$ as

$$
R_{\min } \equiv \inf \left\{a \in \mathbb{R}^{+}: \sum_{l=0}^{\infty} \sum_{m=-l}^{l} \frac{\left|a_{l, m}\right|^{2}}{\sigma_{l}^{2}(a)}<\infty\right\} \text {, }
$$

where inf means “infimum".
The following result follows from (16) and the asymptotic property (12).

\section{Main Result 1. One has}

$$
\begin{gathered}
R_{\min }=\sup \left\{a \in \mathbb{R}^{+}: \lim _{l \rightarrow \infty} \frac{\sqrt{2 l+3} \Gamma(l+3 / 2)}{(k a / 2)^{l}}\left|a_{l, m}\right| \neq 0,\right. \\
\text { at least one } m\},
\end{gathered}
$$

where sup means "supremum".

Proof. Two possibilities arise which explain the use of "inf" (infimum) in (16) instead of just "minimum". In particular, the region of convergence may be closed or open. If it is closed, then condition (16),

$$
\sum_{l=0}^{\infty} \sum_{m=-l}^{l} \frac{\left|a_{l, m}\right|^{2}}{\sigma_{l}^{2}(a)}<\infty
$$

holds for radius $a \geq R_{\min }$ while, if it is open, condition (18) holds for radius $a>R_{\min }$.

Let us consider first the case of a closed region of convergence ( $a \geq R_{\min }$ ). According to the D'Alembert ratio test and using the asymptotics (12), convergence at $a=R_{\min }$ implies

$$
\begin{aligned}
\lim _{l \rightarrow \infty} \frac{\left|a_{l+1, m}\right|^{2} \sigma_{l}^{2}\left(R_{\min }\right)}{\left|a_{l, m}\right|^{2} \sigma_{l+1}^{2}\left(R_{\min }\right)} \\
\quad=\lim _{l \rightarrow \infty} \frac{\left|a_{l+1, m}\right|^{2}}{\left|a_{l, m}\right|^{2}} \frac{(2 l+3)(2 l+5)}{\left(k R_{\min }\right)^{2}} \leq 1
\end{aligned}
$$

while divergence for $a<R_{\min }$,

$$
\sum_{l=0}^{\infty} \sum_{m=-l}^{l} \frac{\left|a_{l, m}\right|^{2}}{\sigma_{l}^{2}(a)}=\infty, \quad a<R_{\min }
$$

implies that at least for some $m$ the associated $l$-indexed sum diverges so that

$$
\begin{aligned}
\lim _{l \rightarrow \infty} & \frac{\left|a_{l+1, m}\right|^{2} \sigma_{l}^{2}(a)}{\left|a_{l, m}\right|^{2} \sigma_{l+1}^{2}(a)} \\
& =\lim _{l \rightarrow \infty} \frac{\left|a_{l+1, m}\right|^{2}}{\left|a_{l, m}\right|^{2}} \frac{(2 l+3)(2 l+5)}{(k a)^{2}} \geq 1
\end{aligned}
$$

at least one $m, \quad a<R_{\min }$.

Since in (21) a can be arbitrarily close to $R_{\min }$ and the $(k a)^{2}$ factor depends continuously on $a$, then, according to (19), (21) necessarily

$$
\lim _{l \rightarrow \infty} \frac{\left|a_{l+1, m}\right|^{2} \sigma_{l}^{2}\left(R_{\min }\right)}{\left|a_{l, m}\right|^{2} \sigma_{l+1}^{2}\left(R_{\min }\right)}=1 \quad \text { at least one } m
$$


and furthermore

$$
\lim _{l \rightarrow \infty} \frac{\left|a_{l+1, m}\right|^{2} \sigma_{l}^{2}(a)}{\left|a_{l, m}\right|^{2} \sigma_{l+1}^{2}(a)}>1 \quad \text { at least one } m, \quad a<R_{\min }
$$

so that necessarily

$$
\lim _{l \rightarrow \infty} \frac{\left|a_{l, m}\right|^{2}}{\sigma_{l}^{2}(a)} \neq 0 \quad \text { at least one } m, \quad a<R_{\min }
$$

which gives the desired result

$$
\lim _{l \rightarrow \infty} \frac{\left|a_{l, m}\right|}{\sigma_{l}(a)} \neq 0 \quad \text { at least one } m, \quad a<R_{\min } .
$$

If on the other hand the region of convergence is open, then

$$
\sum_{l=0}^{\infty} \sum_{m=-l}^{l} \frac{\left|a_{l, m}\right|^{2}}{\sigma_{l}^{2}\left(R_{\min }\right)}=\infty
$$

so that

$$
\lim _{l \rightarrow \infty} \frac{\left|a_{l+1, m}\right|^{2} \sigma_{l}^{2}\left(R_{\min }\right)}{\left|a_{l, m}\right|^{2} \sigma_{l+1}^{2}\left(R_{\min }\right)} \geq 1 \quad \text { at least one } m
$$

which by considerations analogous to those employed above gives (23) and in turn (25). It follows that the condition in (25) can be used to define $R_{\min }$ as is done in (17) where we have also borrowed from the asymptotics in (12).

2.4. Computation Based on Multiple Origins. The proposed multipole radiation theory approach to estimate the minimum convex source region then consists of the following steps: (1) consider a number $n$ of origins of coordinates, $O_{\alpha}$, $\alpha=1,2, \ldots, n$, within the region of interest. (2) For each origin, compute via the test (17) the associated minimum radius $R_{\min }\left(O_{\alpha}\right)$ and associated minimum spherical volume $B_{\text {min }}\left(O_{\alpha}\right)=\left\{\mathbf{r} \in \mathbb{R}^{3}:\left|\mathbf{r}-O_{\alpha}\right| \leq R_{\min }\left(O_{\alpha}\right)\right\}$. (3) The intersection of the regions $B_{\min }\left(O_{\alpha}\right), \alpha=1,2, \ldots, n$ defines a region $\mathscr{B}_{\text {min }}$ bounding the minimum convex source region $B_{\text {min,conv }}$ in the sense that $B_{\text {min,conv }} \subseteq \mathscr{B}_{\text {min }}$. The estimate $\mathscr{B}_{\text {min }}$ becomes closer to $B_{\text {min,conv }}$ as one takes more sample origins and their corresponding minimum spherical volumes. For far origins the surface of the minimum spherical volume behaves locally as a plane, and thus, for example, taking all the points in a sufficiently large sphere as origins and computing their minimum spherical volumes, one obtains the minimum convex source region $B_{\text {min,conv }}$.

\section{Diffraction-Based Analysis}

Next we develop the companion diffraction form of the theory and algorithms for support estimation. In the diffraction point of view, one focuses on the propagation of fields outside the source from a given region (e.g., a closed surface) to another (e.g., another closed surface). There are two versions of this approach. One is useful for the estimation of the minimum convex source region of a far field, and the other is the key for the estimation of the true minimum source region, which can be possibly nonconvex and which lies inside the minimum convex source region. In particular, in the context of spherical regions, which allows application of solutions of the Helmholtz equation in spherical coordinates or in multipole domain, we consider two situations. One is the exterior inverse diffraction problem consisting of (back) propagation of fields that are known for $r \geq a$ into a spherical surface of radius $b<a$ centered about the same origin. The other situation is one involving an interior inverse diffraction problem, where one (back) propagates fields that are known in the interior of a given spherical volume of radius $b$ (for $r \leq b)$ to a spherical surface of radius $a>b$ centered about the same origin. Solutions associated to the first problem yield a method for computing bounds for the minimum convex source region of a far field while solutions to the second problem provide a method to sharpen the estimate of the minimum source region by refining the nonconvex boundaries of the minimum source region.

In this section we establish the diffraction-based algorithm for the minimum convex source region. We find that it is equivalent to the one based on the radiationbased approach discussed earlier. In the next section we develop the companion diffraction-based algorithm for the possibly nonconvex minimum source region. The respective radiation-based nonconvex support inversion method is not discussed in the paper since, as its convex counterpart, it gives in the end the same algorithm.

3.1. Forward Problem. According to (2), the field at the sphere defined by $r=b>R$ is given by

$$
\psi_{b}(\widehat{\mathbf{r}}) \equiv \psi(b \widehat{\mathbf{r}})=i k \sum_{l, m} a_{l, m} h_{l}^{(1)}(k b) Y_{l, m}(\widehat{\mathbf{r}}) .
$$

It follows from (28) and the orthonormality of the spherical harmonics that

$$
\widehat{b}_{l, m} \equiv \int_{S^{2}} d \widehat{\mathbf{r}} Y_{l, m}^{*}(\widehat{\mathbf{r}}) \psi_{b}(\widehat{\mathbf{r}})=i k a_{l, m} h_{l}^{(1)}(k b) .
$$

Then the mapping from the field at $r=b$, in particular,

$$
\psi_{b}(\widehat{\mathbf{r}})=\sum_{l, m} \widehat{b}_{l, m} Y_{l, m}(\widehat{\mathbf{r}}),
$$

to the multipole moments $a_{l, m}$, which are related to the far field radiation pattern via (6), (7), is characterized by the relation

$$
a_{l, m}=\frac{\widehat{b}_{l, m}}{\left[i k h_{l}^{(1)}(k b)\right]} \text {. }
$$

From the asymptotic relation (see [3, page 77])

$$
h_{l}^{(1)}(k r) \sim-i \frac{\Gamma(l+1 / 2)}{2 \sqrt{\pi}}\left(\frac{2}{k r}\right)^{l+1}, \quad l \gg k r,
$$

we get $h_{l}^{(1)}(k b) \rightarrow \infty$ as $l \rightarrow \infty$ so that from (31) $a_{l, m} \rightarrow 0$ as $l \rightarrow \infty$. Thus the fine details of the field at $r=b$ are 
not contained in the far field, which is the reason behind the ill-posedness of the associated inverse diffraction problem of reconstructing the near field at $r=b$ from the far field. Furthermore, using the rule of thumb $\left[h_{l}^{(1)}(k b)\right]^{-1} \simeq 0$ for $l \gtrsim k b$, then

$$
\begin{aligned}
& a_{l, m}=\frac{\widehat{b}_{l, m}}{\left[i k h_{l}^{(1)}(k b)\right]}, \quad l \lesssim k b, \\
& a_{l, m} \simeq 0, \quad l \gtrsim k b ;
\end{aligned}
$$

hence only the fine details of the near field at $r=b$ up to expansion index $l \simeq k b$ are visible in the far zone, under noise or perturbations.

It also follows from (2), (28), (29), (30), and (31) that the more general forward mapping from the field $\psi_{b}$ at $r=b\left(\psi_{b}\right)$ to the field $\psi_{a}$ at $r=a>b$ where

$$
\psi_{a}(\widehat{\mathbf{r}}) \equiv \psi(a \widehat{\mathbf{r}})=\sum_{l, m} \widehat{a}_{l, m} Y_{l, m}(\widehat{\mathbf{r}})
$$

is described by

$$
\widehat{a}_{l, m}=\left[\frac{h_{l}^{(1)}(k a)}{h_{l}^{(1)}(k b)}\right] \widehat{b}_{l, m} .
$$

3.2. Inverse Problem. Consider now the inverse diffraction problem of estimating $\psi_{b}$ from knowledge of $\psi_{a}$ where $b<$ $a$. Equivalently $\widehat{a}_{l, m}$ for $r=a>b$ is given (measured or specified), and the problem consists of estimating $\widehat{b}_{l, m}$ for $r=b<a$ assuming that the field obeys the homogeneous Helmholtz equation for $r>b$ and the radiation condition at infinity. Then inverting (35),

$$
\widehat{b}_{l, m}=\left[\frac{h_{l}^{(1)}(k b)}{h_{l}^{(1)}(k a)}\right] \widehat{a}_{l, m} .
$$

The field at $r=b<a$ is given by (30) and is square-integrable if it obeys the Picard condition:

$$
\begin{aligned}
\left\|\psi_{b}\right\|^{2} & =\int_{S^{2}} d \widehat{\mathbf{r}}\left|\psi_{b}(\widehat{\mathbf{r}})\right|^{2}=\sum_{l, m}\left|\widehat{b}_{l, m}\right|^{2} \\
& =\sum_{l, m}\left|\widehat{a}_{l, m}\right|^{2}\left[\frac{h_{l}^{(1)}(k b)}{h_{l}^{(1)}(k a)}\right]^{2}<\infty
\end{aligned}
$$

which by recalling the asymptotic relation (32) requires for $L^{2}$ convergence

$$
\lim _{l \rightarrow \infty}\left(\frac{a}{b}\right)^{l}\left|\widehat{a}_{l, m}\right|=0, \quad b<a,
$$

and furthermore one can show that, if the Picard condition does not hold, then

$$
\lim _{l \rightarrow \infty}\left(\frac{a}{b}\right)^{l}\left|\widehat{a}_{l, m}\right| \neq 0, \quad b<a, \quad \text { at least one } m .
$$

This result applies in both near and far zones and is the key for the respective extensions to the near zone. In the far zone case, for $a \rightarrow \infty$, we use the far field results associated to (30) and (31), obtaining that the $L^{2}$ requirement for the reconstructed field or Picard condition becomes

$$
\sum_{l, m}\left|a_{l, m}\right|^{2}\left|h_{l}^{(1)}(k b)\right|^{2}<\infty
$$

which requires for convergence

$$
\lim _{l \rightarrow \infty}\left|h_{l}^{(1)}(k b)\right|\left|a_{l, m}\right|=0,
$$

which in view of (32) gives

$$
\lim _{l \rightarrow \infty} \Gamma\left(l+\frac{1}{2}\right)\left(\frac{2}{k b}\right)^{l}\left|a_{l, m}\right|=0 .
$$

Moreover, if the Picard condition does not hold,

$$
\lim _{l \rightarrow \infty} \Gamma\left(l+\frac{1}{2}\right)\left(\frac{2}{k b}\right)^{l}\left|a_{l, m}\right| \neq 0 \quad \text { at least one } m,
$$

and we arrive through arguments analogous to those leading to main result 1 to the following result whose proof is omitted for economy.

Main Result 2. One has

$$
\begin{gathered}
R_{\min }=\sup \left\{b \in \mathbb{R}^{+}: \lim _{l \rightarrow \infty} \frac{\Gamma(l+1 / 2)}{(k b / 2)^{l}}\left|a_{l, m}\right| \neq 0,\right. \\
\text { at least one } m\} .
\end{gathered}
$$

Equation (44) is the diffraction-based counterpart of the result (17) discussed earlier in the radiation-based approach. Furthermore, clearly if

$$
\lim _{l \rightarrow \infty} \frac{\Gamma(l+1 / 2)\left|a_{l, m}\right|}{(k a / 2)^{l}} \neq 0,
$$

then also

$$
\lim _{l \rightarrow \infty} \frac{\sqrt{2 l+3} \Gamma(l+3 / 2)\left|a_{l, m}\right|}{(k a / 2)^{l}} \neq 0 .
$$

In addition, clearly if

$$
\lim _{l \rightarrow \infty} \frac{\sqrt{2 l+3} \Gamma(l+3 / 2)\left|a_{l, m}\right|}{(k a / 2)^{l}} \neq 0,
$$

then also

$$
\lim _{l \rightarrow \infty} \frac{\Gamma(l+1 / 2)\left|a_{l, m}\right|}{\left(k a^{\prime} / 2\right)^{l}} \neq 0, \quad a^{\prime}<a .
$$

As a consequence, the value of $R_{\text {min }}$ defined by main results 1 and 2 (see (17) and (44)) is in fact the same. Thus the respective diffraction-based algorithm to estimate the minimum convex source region is the same outlined in Section 2.4.

Here we also wish to point out that the Picard condition adopted above is quite fundamental, as it is germane in defining the class of realizable fields not only in the $L^{2}$ sense but also in the uniform and absolute convergence senses. This 
can be shown for the diffraction-based approach from a result in $[3$, thm. 12 , page 77$]$.

\section{Estimation of the (Possibly Nonconvex) Minimum Source Region}

In order to sharpen the estimate of the minimum source region, including nonconvex regions, we must consider forward and inverse diffraction problems in the near field, associated to a complementary geometry where the field data are known in the interior of a given spherical volume (interior data) and the field is backpropagated into outer concentric spheres. If the far field is known in closed form, then it is theoretically possible to estimate also the associated near field. If, on the other hand, the data are experimental or computational and thus subject to noise and perturbations, then the near field can be estimated only up to an essentially finitedimensional representation as outlined in the discussion of (33).

First we review the radiation problem from a perspective that complements our discussion in Section 2.1 and introduces the forward and inverse diffraction problems leading to the sought-after method to extract nonconvex source support information. It is well known that the solution of (1) that obeys Sommerfeld's radiation condition can be expressed as the Green function integral:

$$
\psi(\mathbf{r})=\int d \mathbf{r}^{\prime} G\left(\mathbf{r}, \mathbf{r}^{\prime}\right) \rho\left(\mathbf{r}^{\prime}\right)
$$

where the outgoing wave Green function

$$
G\left(\mathbf{r}, \mathbf{r}^{\prime}\right)=\frac{e^{i k\left|\mathbf{r}-\mathbf{r}^{\prime}\right|}}{4 \pi\left|\mathbf{r}-\mathbf{r}^{\prime}\right|}
$$

Furthermore, in view of the addition theorem for spherical Hankel functions (see [7, page 428]),

$$
\begin{aligned}
& G\left(\mathbf{r}, \mathbf{r}^{\prime}\right) \\
& \quad=i k \sum_{l=0}^{\infty} \sum_{m=-l}^{l} j_{l}\left(k r_{<}\right) h_{l}^{(1)}\left(k r_{>}\right) Y_{l, m}(\widehat{\mathbf{r}}) Y_{l, m}^{*}\left(\widehat{\mathbf{r}}^{\prime}\right),
\end{aligned}
$$

where $r_{<}=\min \left(r, r^{\prime}\right)$ (where $\left.r^{\prime} \equiv\left|\mathbf{r}^{\prime}\right|\right)$ and $r_{>}=\max \left(r, r^{\prime}\right)$.

The result (51) holds for any choice of the origin of coordinates. Thus if, for one origin, $O$, we denote in spherical coordinates $\mathbf{r}=(r, \theta, \phi)$ and $\widehat{\mathbf{r}}=(\theta, \phi)$, and $\mathbf{r}^{\prime}=\left(r^{\prime}, \theta^{\prime}, \phi^{\prime}\right)$ and $\widehat{\mathbf{r}}^{\prime}=\left(\theta^{\prime}, \phi^{\prime}\right)$, while for another origin, $O^{\prime}$, we use $\mathbf{r}=$ $\left(r_{O^{\prime}}, \theta_{O^{\prime}}, \phi_{O^{\prime}}\right)$ and $\widehat{\mathbf{r}}_{O^{\prime}}=\left(\theta_{O^{\prime}}, \phi_{O^{\prime}}\right)$, and $\mathbf{r}^{\prime}=\left(r_{O^{\prime}}^{\prime}, \theta_{O^{\prime}}, \phi_{O^{\prime}}^{\prime}\right)$ and $\widehat{\mathbf{r}}^{\prime}=\left(\theta_{\mathrm{O}^{\prime}}^{\prime}, \phi_{\mathrm{O}^{\prime}}^{\prime}\right)$, then (51) holds and also

$$
\begin{aligned}
& G\left(\mathbf{r}, \mathbf{r}^{\prime}\right) \\
& \quad=i k \sum_{l=0}^{\infty} \sum_{m=-l}^{l} j_{l}\left(k r_{<, O^{\prime}}\right) h_{l}^{(1)}\left(k r_{>, O^{\prime}}\right) Y_{l, m}\left(\widehat{\mathbf{r}}_{O^{\prime}}\right) Y_{l, m}^{*}\left(\widehat{\mathbf{r}}_{\mathrm{O}^{\prime}}^{\prime}\right),
\end{aligned}
$$

where $r_{<, O^{\prime}}=\min \left(r_{O^{\prime}}, r_{O^{\prime}}^{\prime}\right)$ and $r_{>, O^{\prime}}=\max \left(r_{O^{\prime}}, r_{O^{\prime}}^{\prime}\right)$. It is possible to choose the coordinate systems $O$ and $O^{\prime}$ such that

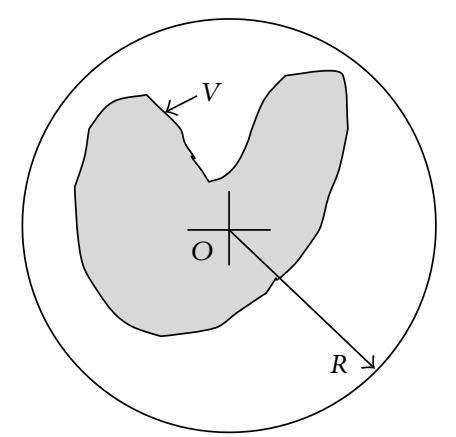

FIGURE 3: Illustration of an origin $O$ for which the support of the source $\rho$ is confined within a ball of radius $R$ centered at this origin.

$r_{<}=r^{\prime}$ while $r_{<, O^{\prime}}=r_{\mathrm{O}^{\prime}}$ in which case the results (51) and (52) give

$$
\begin{aligned}
& \sum_{l=0}^{\infty} \sum_{m=-l}^{l} j_{l}\left(k r^{\prime}\right) h_{l}^{(1)}(k r) Y_{l, m}(\widehat{\mathbf{r}}) Y_{l, m}^{*}\left(\widehat{\mathbf{r}}^{\prime}\right) \\
& \quad=\sum_{l=0}^{\infty} \sum_{m=-l}^{l} j_{l}\left(k r_{O^{\prime}}\right) h_{l}^{(1)}\left(k r_{O^{\prime}}^{\prime}\right) Y_{l, m}\left(\widehat{\mathbf{r}}_{O^{\prime}}\right) Y_{l, m}^{*}\left(\widehat{\mathbf{r}}_{O^{\prime}}^{\prime}\right) .
\end{aligned}
$$

This result is useful to show that the minimum source region of a set of point sources is the set of the point source locations.

For a source $\rho$ whose support $V$ is confined inside a ball of radius $R$ centered about the origin $O$ (see Figure 3 ), we obtain from (49) and (51) the result (2) with $a_{l, m}$ given by (3) which defines the field for $r>R$, as expected. Furthermore, since there exists a source inside the minimum source region $V_{\min } \subseteq V$ that produces the given far field (see (9)) and thereby also the same multipole moments $a_{l, m}$, it follows that we can also apply (49) and (51) to this source, obtaining

$$
\psi(\mathbf{r})=i k \sum_{l=0}^{\infty} \sum_{m=-l}^{l} a_{l, m} h_{l}^{(1)}(k r) Y_{l, m}(\widehat{\mathbf{r}}), \quad r>R_{\min },
$$

where $R_{\min }$ is the radius of the smallest spherical volume that is centered about the same origin and contains the minimum source region $V_{\min } \subseteq V$ of the given far field, as is illustrated in Figure 4. Expression (54) is implicit in the diffraction-based inverse support approach of Section 3. The result (54) holds for any origin $O$. The intersection of the minimum spherical source regions $B_{\min }=\left\{\mathbf{r} \in \mathbb{R}^{3}: r \leq\right.$ $\left.R_{\min }\right\}$ for different origins establishes a convex region where the minimum source region resides, and it is theoretically possible for sufficiently many such origins to actually define the minimum convex source region $B_{\text {min,conv }}$ of the given far field, which is defined by the convex hull of the minimum source region $V_{\min }$ and which is contained in the convex hull of the original source's support $V$. Now, since (54) holds for any origin, this also means that we can compute the radiated field (due to any source inside the minimum source region that generates the given far field) everywhere outside the minimum convex source region. Once this is done we can 


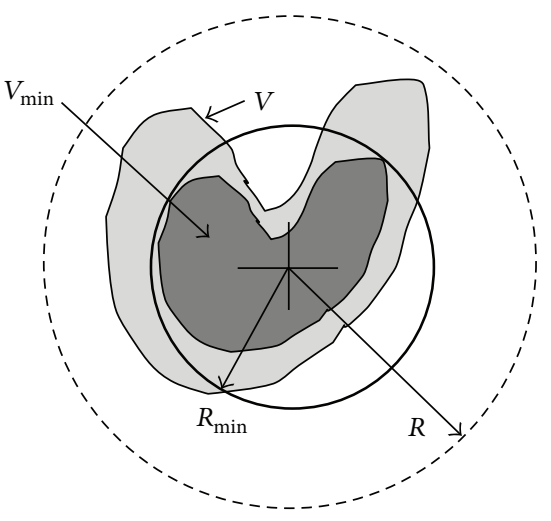

FIgURE 4: This figure expands the interpretation of Figure 3. It illustrates the relations between $V_{\min }$ and $V$, and $R_{\min }$ and $R$, for the same source and origin shown in Figure 3.

use this information to refine our estimate of the minimum source region beyond the minimum convex source region by extracting the respective nonconvex support information as we explain next.

To extract the desired nonconvex support information we note that, if, on the other hand, the origin of coordinates $O$ is such that $V$ is contained outside a ball of radius $R^{\text {int }}$ centered at the origin (see Figure 5), then the field in the interior of this circle of radius $R^{\text {int }}$ is given from (49) and (51) by

$$
\psi(\mathbf{r})=i k \sum_{l=0}^{\infty} \sum_{m=-l}^{l} g_{l, m} j_{l}(k r) Y_{l, m}(\widehat{\mathbf{r}}), \quad r<R^{\mathrm{int}},
$$

where

$$
g_{l, m}=\int_{R^{\text {int }}}^{\infty} d r r^{2} h_{l}^{(1)}(k r) \int_{S^{2}} d \widehat{\mathbf{r}} Y_{l, m}^{*}(\widehat{\mathbf{r}}) \rho(\mathbf{r}) .
$$

Furthermore, since there exists a source inside the minimum source region that produces the given far field and thereby also the same field outside $V$ as the original source $\rho$, it follows that by applying (49), (51) to the source inside $V_{\min }$ while using (53), (55), (56) we obtain

$$
\psi(\mathbf{r})=i k \sum_{l=0}^{\infty} \sum_{m=-l}^{l} g_{l, m} j_{l}(k r) Y_{l, m}(\widehat{\mathbf{r}}), \quad r<R_{\min }^{\mathrm{int}}
$$

where the critical radius $R_{\min }^{\mathrm{int}}$ is the radius of the largest spherical volume centered about the same origin that is disjoint to the minimum source region $V_{\min }$ and is in fact tangential to it in a way that allows the extraction of its nonconvex content as is illustrated in Figures 6, 7, and 8 . We detail in the following the methodology to compute this critical radius $R_{\min }^{\text {int }}$ and elaborate the method to extract nonconvex support information about the minimum source region.

So far (in the two previous sections) we have derived a methodology to estimate the minimum convex source region $B_{\text {min,conv }}$. We also know from the discussion following (54) that it is theoretically possible to estimate the field everywhere outside the minimum convex source region from

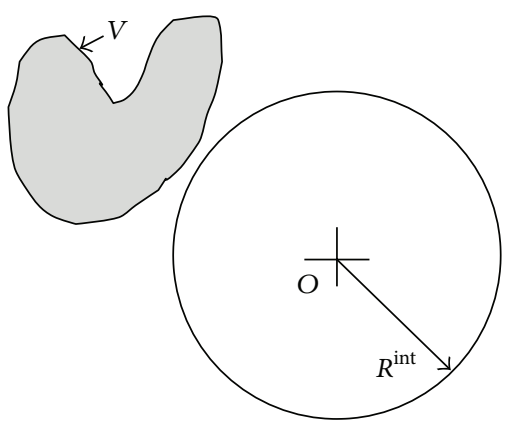

FIGURE 5: Illustration of an origin $O$ for which the support of the source $\rho$ is contained outside a ball of radius $R^{\text {int }}$ centered at this origin.

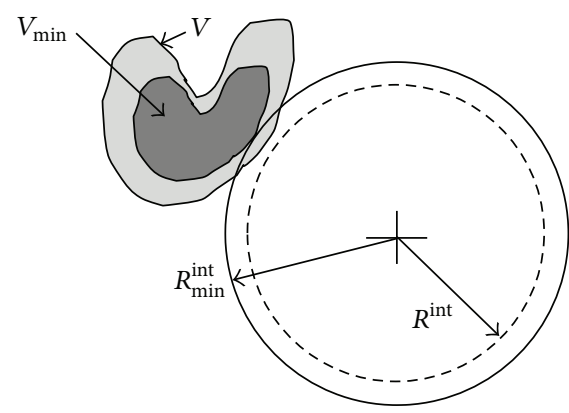

FIgURE 6: This figure expands the interpretation of Figure 5. It illustrates the relations between $V_{\min }$ and $V$, and $R_{\min }^{\text {int }}$ and $R^{\text {int }}$, for the same source and origin shown in Figure 5.

the knowledge of the far field or the multipole moments. To estimate the minimum source region $V_{\min }$ we will backpropagate the fields in the interior of a spherical volume of radius $R$ that is centered at the origin $O$ up to the critical sphere of radius $R_{\min }^{\text {int }}$ beyond which such backpropagation is not possible (since for larger radius the backpropagated field is not $L^{2}$ ). Clearly the spherical volumes whose fields will be backpropagated must be such that their interior fields are known (they can be computed from the far field). Therefore we pick first spherical volumes of center $O$ and radius $R^{\text {int }}$ that are entirely localized outside the derived minimum convex source region (see Figure 7) since their interior fields can be computed via (54) from the far field or the multipole moments. Subsequently, as the respective backpropagations are carried out into concave boundaries of the minimum source region, we will as a by product also compute the backpropagated fields up to those boundaries, so that we will be able to choose spherical volumes of center $O$ and radius $R^{\text {int }}$ intersecting the minimum convex source region but outside the best current estimate of the boundary of the minimum source region. The process is illustrated in Figure 8. In the discussion that follows, it is implicitly assumed that the field in the interior of a spherical region of radius $b$ centered at the origin has been estimated this way from previous computation steps, and the goal is to compute the radius $R_{\min }^{\mathrm{int}}$ of the largest sphere up to which the available exterior radiated field can be backpropagated, which defines 


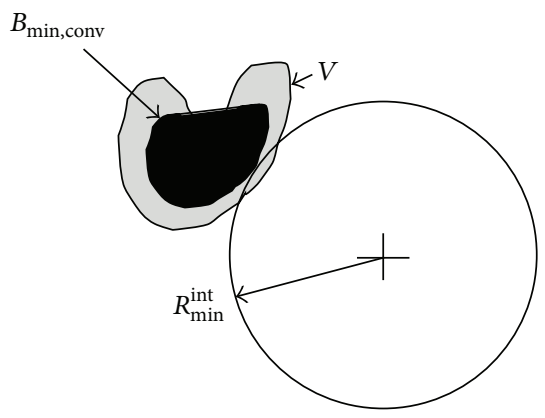

FIGURE 7: Illustration of the minimum convex source region $B_{\text {min,conv }}$ corresponding to the minimum source region $V_{\min }$ in Figure 6. The radiated field can be estimated everywhere outside $B_{\text {min,conv }}$, and this information can be used subsequently to estimate the critical radius $R_{\text {min }}^{\text {int }}$ of the largest sphere up to which the known radiated field can be backpropagated, which establishes a boundary for the minimum source region $V_{\min }$. The geometry of this backpropagation pierces or intersects concave portions of $B_{\text {min,conv }}$, allowing the extraction of the pending nonconvex support information of $V_{\min }$ that is not contained in $B_{\text {min,conv }}$.

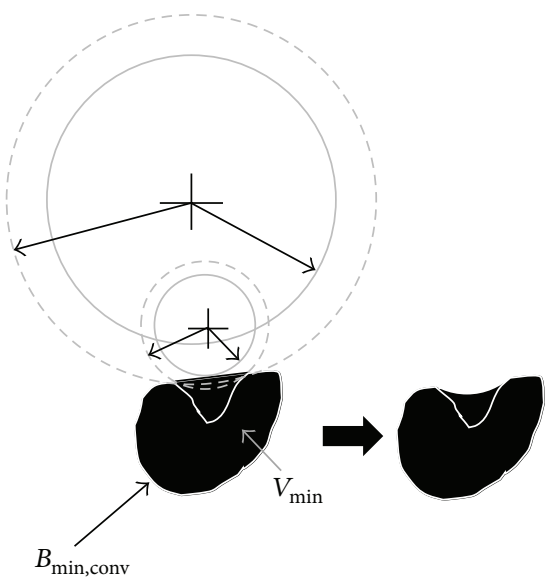

FIGURE 8: Successive application of the diffraction-based estimation of the critical sphere of radius $R_{\text {min }}^{\text {int }}$, based on field information available from previous iterations. This method allows the estimation of concave boundaries of the minimum source region. Thus it allows, in theory, the estimation of the minimum source region $V_{\min }$ which can be nonconvex.

the possibly nonconvex boundary of the minimum source region.

4.1. Forward Problem. In view of (57) it follows that if the field is prescribed at the sphere defined by $r=a<R_{\min }^{\text {int }}$, in particular,

$$
\psi_{a}(\widehat{\mathbf{r}})=\psi(a \widehat{\mathbf{r}})=\sum_{l, m} \bar{a}_{l, m} Y_{l, m}(\widehat{\mathbf{r}}), \quad a<R_{\min }^{\mathrm{int}},
$$

where

$$
\bar{a}_{l, m}=g_{l, m} j_{l}(k a)
$$

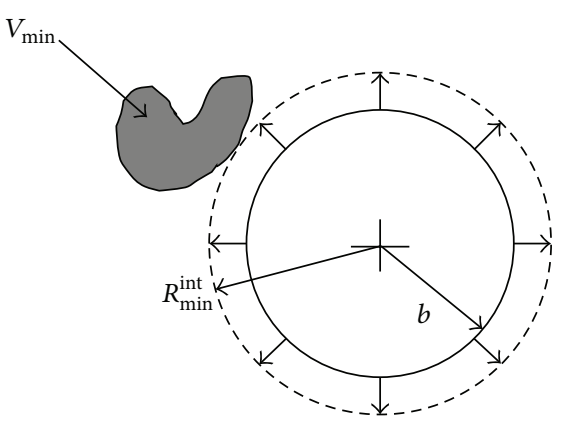

FIGURE 9: Backpropagation of the field at the sphere of radius $r=$ $b$ to the respective field at a larger concentric sphere. This process breaks down at the critical radius $R_{\min }^{\text {int }}$ which defines the soughtafter boundary of the minimum source region $V_{\min }$.

then the field $\psi_{b}(\widehat{\mathbf{r}}) \equiv \psi(b \widehat{\mathbf{r}})$ for any $r=b<a$ can be computed from $\psi_{a}$ or equivalently $\bar{a}_{l, m}$ via

$$
\psi_{b}(\widehat{\mathbf{r}})=\sum_{l, m} \bar{b}_{l, m} Y_{l, m}(\widehat{\mathbf{r}}),
$$

where

$$
\bar{b}_{l, m}=\bar{a}_{l, m} \frac{j_{l}(k b)}{j_{l}(k a)}
$$

which in view of (11) gives

$$
\lim _{l \rightarrow \infty} \frac{\bar{b}_{l, m}}{\overline{\bar{l}}_{l, m}}=\left(\frac{b}{a}\right)^{l} .
$$

4.2. Inverse Problem. Consider now the interior inverse diffraction problem of reconstructing the field at $r=a\left(\psi_{a}\right)$ from knowledge of the field for $r \leq b<a\left(\psi_{b}\right)$ (see Figure 9). We obtain

$$
\psi_{a}(\widehat{\mathbf{r}})=\sum_{l, m} \bar{b}_{l, m}\left[\frac{j_{l}(k a)}{j_{l}(k b)}\right] Y_{l, m}(\widehat{\mathbf{r}})
$$

which by requiring square-integrability of the solution gives

$$
\sum_{l, m}\left|\bar{b}_{l, m}\right|^{2}\left[\frac{j_{l}(k a)}{j_{l}(k b)}\right]^{2}<\infty
$$

On the other hand, if it is not possible to find an $L^{2}$ field at $\psi_{a}$ capable of producing the given field $\psi$ for $r \leq b<a$, then necessarily the series in (64) diverges, which can be shown to imply

$$
\lim _{l \rightarrow \infty}\left|\bar{b}_{l, m}\right|\left[\frac{j_{l}(k a)}{j_{l}(k b)}\right] \neq 0, \quad \text { at least one } m .
$$

Using the asymptotic result (11), this implies

$$
\lim _{l \rightarrow \infty}\left|\bar{b}_{l, m}\right|\left(\frac{a}{b}\right)^{l} \neq 0, \quad \text { at least one } m .
$$


Equivalently, we can rewrite (65) and (66) as

$$
\lim _{l \rightarrow \infty}\left|j_{l}(k a)\right|\left|g_{l, m}\right| \neq 0, \quad \text { at least one } m \text {. }
$$

Thus from (11),

$$
\lim _{l \rightarrow \infty}\left(\frac{k a}{2}\right)^{l}\left[\Gamma\left(l+\frac{3}{2}\right)\right]^{-1}\left|g_{l, m}\right| \neq 0, \quad \text { at least one } m \text {. }
$$

The radius $R_{\min }^{\mathrm{int}}$, defining the boundary of the (generally nonconvex) minimum source region, is then given by the infimum value of $a$ such that (66) and (68) hold. We summarize this result as follows.

Main Result 3. One has

$$
\begin{gathered}
R_{\min }^{\text {int }}=\inf \left\{a \in \mathbb{R}^{+}: \lim _{l \rightarrow \infty}\left(\frac{k a}{2}\right)^{l}\left[\Gamma\left(l+\frac{3}{2}\right)\right]^{-1}\left|g_{l, m}\right| \neq 0,\right. \\
\text { at least one } m\} .
\end{gathered}
$$

4.3. Algorithm. The method to estimate the minimum source region now takes complete form as follows.

(1) Applying the exterior inverse diffraction approach of the previous subsection, compute the convex region $\mathscr{B}_{\text {min }}$ where $V_{\text {min }} \subseteq B_{\text {min,conv }} \subseteq \mathscr{B}_{\text {min }}$. For sufficiently many computational origins, it is possible that the estimate $\mathscr{B}_{\text {min }}=B_{\text {min,conv }}$.

(2) Consider next reference origins outside $\mathscr{B}_{\min }$, say $\mathrm{O}_{\alpha}$, $\alpha=1,2, \ldots, n$, and evaluate the field (via the exterior inverse diffraction approach) over spheres centered about those origins and having radius as large as possible but not intersecting the volume $\mathscr{B}_{\text {min }}$. Let us assume such computational surfaces correspond to radii $b_{\alpha}$, with centers $O_{\alpha}$.

(3) Using the value of the field on such surfaces, as computed via exterior inverse diffraction from far field data, compute the respective minimum radius $R_{\min }^{\mathrm{int}}(\alpha)$.

(4) Compute the union $U^{\text {int }}$ of all the regions $B_{\min }^{\text {int }}(\alpha)=$ $\left\{\mathbf{r} \in \mathbb{R}^{3}:\left|\mathbf{r}-O_{\alpha}\right| \leq R_{\min }^{\mathrm{int}}(\alpha)\right\}$. Compute the respective complement $\bar{U}^{\text {int }}=\left\{\mathbf{r} \in \mathbb{R}^{3}: \mathbf{r} \notin U^{\text {int }}\right\}$.

(5) Compute the intersection of $\bar{U}^{\text {int }}$ and $\mathscr{B}_{\text {min }}$, which defines the revised estimate $V_{\text {min,est }} \supseteq V_{\text {min }}$ for the minimum source region which can be nonconvex, in particular,

$$
V_{\text {min,est }}=\mathscr{B}_{\min } \cap \bar{U}^{\text {int }} \text {. }
$$

(6) The information used in the previous steps is the field outside the convex region $\mathscr{B}_{\min }$. However, as the estimate $V_{\text {min,est }}$ becomes closer to $V_{\text {min }}$ than $\mathscr{B}_{\text {min }}$, we need to repeat steps (2)-(4) for computational surfaces located outside the best current estimate $V_{\text {min,est }}$, whose respective fields for the required backpropagation are computed via the interior inverse diffraction approach explained in this section. The counterpart of step (5) associated to the second and following iterations is $V_{\text {min,est }}$ ("current") = $V_{\text {min,est }}($ "previous" $) \cap \bar{U}^{\text {int }}$. By continuing this process (see Figure 8), it is theoretically possible to obtain $V_{\text {min,est }}=V_{\min }$, as desired.

\section{Theory and Algorithms in 2D Space}

We consider next the $2 \mathrm{D}$ version of the theory for the Helmholtz operator in 2D space. To tie the results to electromagnetic applications, we consider radiation and scattering problems involving transverse magnetic $z\left(\mathrm{TM}^{z}\right)$ polarization for which radiation is governed by the $2 \mathrm{D}$ Helmholtz equation

$$
\left(\nabla^{2}+k^{2}\right) E_{z}(\mathbf{r})=-i \omega \mu I_{z}(\mathbf{r})
$$

where the wavenumber

$$
k=\omega \sqrt{\mu \epsilon}
$$

where $\omega$ is the angular oscillation frequency, $\epsilon$ is the free space permittivity, and $\mu$ is the free space permeability and where $E_{z}$ is the $z$ component of the electric field and $I_{z}$ is the $z$ component of the current distribution that produces the field. The radiated field is well known to be given by

$$
\begin{aligned}
E_{z}(\mathbf{r}) & =-i \omega \mu \int d \mathbf{r}^{\prime} G\left(\mathbf{r}, \mathbf{r}^{\prime}\right) I_{z}\left(\mathbf{r}^{\prime}\right) \\
& =-\frac{\omega \mu}{4} \int d \mathbf{r}^{\prime} H_{0}^{(1)}\left(k\left|\mathbf{r}-\mathbf{r}^{\prime}\right|\right) I_{z}\left(\mathbf{r}^{\prime}\right),
\end{aligned}
$$

where $G\left(\mathbf{r}, \mathbf{r}^{\prime}\right)$ is the outgoing wave $2 \mathrm{D}$ Green function of the Helmholtz operator and $H_{0}^{(1)}$ is the Hankel function of the first kind and order 0 . From the addition theorem for Hankel functions, we have

$$
\begin{aligned}
H_{0}^{(1)}\left(k\left|\mathbf{r}-\mathbf{r}^{\prime}\right|\right) & =4 i G\left(\mathbf{r}, \mathbf{r}^{\prime}\right) \\
& =\sum_{m=-\infty}^{\infty} J_{m}\left(k \rho_{<}\right) H_{m}^{(1)}\left(k \rho_{>}\right) e^{-i m\left(\phi-\phi^{\prime}\right)},
\end{aligned}
$$

where $\rho_{<}=\min \left(\rho, \rho^{\prime}\right)$ and $\rho_{>}=\max \left(\rho, \rho^{\prime}\right)$ where $\rho$ is the distance from $\mathbf{r}$ to the origin of coordinates and $\rho^{\prime}$ is the distance from $\mathbf{r}^{\prime}$ to the origin. The result (74) holds for any choice of the origin of coordinates. Thus if for one origin, $O$, we denote $\mathbf{r}=(\rho, \phi)$ and $\mathbf{r}^{\prime}=\left(\rho^{\prime}, \phi^{\prime}\right)$, and for another origin, $O^{\prime}, \mathbf{r}=\left(\rho_{O^{\prime}}, \phi_{O^{\prime}}\right)$ and $\mathbf{r}^{\prime}=\left(\rho_{O^{\prime}}^{\prime}, \phi_{O^{\prime}}^{\prime}\right)$, then $(74)$ holds and also

$$
\begin{aligned}
& 4 i G\left(\mathbf{r}, \mathbf{r}^{\prime}\right) \\
& \left.\quad=\sum_{m=-\infty}^{\infty} J_{m}\left(k \rho_{<, O^{\prime}}\right) H_{m}^{(1)}\left(k \rho_{>, O^{\prime}}\right) e^{-i m\left(\phi_{O^{\prime}}-\phi_{O^{\prime}}^{\prime}\right.}\right),
\end{aligned}
$$


where of course $\rho_{<, O^{\prime}}=\min \left(\rho_{O^{\prime}}, \rho_{O^{\prime}}^{\prime}\right)$ and $\rho_{>, O^{\prime}}=$ $\max \left(\rho_{\mathrm{O}^{\prime}}, \rho_{\mathrm{O}^{\prime}}^{\prime}\right)$. It is possible to choose the coordinate systems $O$ and $O^{\prime}$ such that $\rho_{<}=\rho^{\prime}$ while $\rho_{<, O^{\prime}}=\rho_{O^{\prime}}$ in which case the results (74) and (75) give

$$
\begin{aligned}
\sum_{m=-\infty}^{\infty} J_{m}\left(k \rho^{\prime}\right) H_{m}^{(1)}(k \rho) e^{-i m\left(\phi-\phi^{\prime}\right)} & \\
& =\sum_{m=-\infty}^{\infty} J_{m}\left(k \rho_{O^{\prime}}\right) H_{m}^{(1)}\left(k \rho_{O^{\prime}}^{\prime}\right) e^{-i m\left(\phi_{O^{\prime}}-\phi_{O^{\prime}}^{\prime}\right)} .
\end{aligned}
$$

This result is useful to show that the minimum source region of a set of point sources is the set of the point source locations.

For a source whose support $V$ is confined inside a 2-ball or disk of radius $R$ centered about the origin $O$, we have from (73) and (74)

$$
\begin{aligned}
E_{z}(\mathbf{r} & =(\rho, \phi)) \\
& =-\frac{\omega \mu}{4} \sum_{m=-\infty}^{\infty} a_{m} H_{m}^{(1)}(k \rho) e^{-i m \phi}, \quad \rho>R,
\end{aligned}
$$

where

$$
a_{m}=\int_{0}^{R} d \rho^{\prime} \rho^{\prime} \int_{0}^{2 \pi} d \phi^{\prime} J_{m}\left(k \rho^{\prime}\right) e^{i m \phi^{\prime}} I_{z}\left(\rho^{\prime}, \phi^{\prime}\right) .
$$

Using the large argument approximation for the Hankel function

$$
H_{m}^{(1)}(k \rho) \sim \sqrt{\frac{2}{\pi k \rho}} e^{i[k \rho-m(\pi / 2)-\pi / 4]},
$$

we get from (85) the far zone behavior:

$$
E_{z}(\rho, \phi) \sim-\frac{\omega \mu}{4} \sqrt{\frac{2}{\pi k \rho}} e^{i(k \rho-\pi / 4)} f(\phi),
$$

where the far field radiation pattern

$$
f(\phi)=\sum_{m=-\infty}^{\infty} i^{-m} a_{m} e^{-i m \phi}
$$

so that in view of the orthogonality of the complex exponentials for integer $m$

$$
a_{m}=\frac{i^{m}}{2 \pi} \int_{0}^{2 \pi} d \phi e^{i m \phi} f(\phi) .
$$

In addition, it can be shown that there is a source inside the minimum source region $V_{\min } \subseteq V$ that generates the given far field, which is associated to the multipole moments $a_{m}$. By applying the above formulation to this source, we obtain

$$
\begin{aligned}
E_{z}(\mathbf{r} & =(\rho, \phi)) \\
& =-\frac{\omega \mu}{4} \sum_{m=-\infty}^{\infty} a_{m} H_{m}^{(1)}(k \rho) e^{-i m \phi}, \quad \rho>R_{\min },
\end{aligned}
$$

where $R_{\min }$ is the radius of the smallest ball that is centered about the same origin and contains the minimum source region $V_{\min } \subseteq V$ of the given far field. Now, since (83) holds for any origin, this means that we can compute a valid radiated field associated to the given far field everywhere outside the minimum convex source region of the given far field.

On the other hand, if the origin of coordinates $O$ is such that $V$ is contained outside a circle of radius $R^{\text {int }}$ centered at the origin, then the field in the interior of this circle of radius $R^{\text {int }}$ is given from (73) and (74) by

$$
\begin{aligned}
E_{z}(\mathbf{r} & =(\rho, \phi)) \\
& =-\frac{\omega \mu}{4} \sum_{m=-\infty}^{\infty} g_{m} J_{m}(k \rho) e^{-i m \phi}, \quad \rho<R^{\mathrm{int}},
\end{aligned}
$$

where

$$
g_{m}=\int_{R^{\mathrm{int}}}^{\infty} d \rho^{\prime} \rho^{\prime} \int_{0}^{2 \pi} d \phi^{\prime} H_{m}^{(1)}\left(k \rho^{\prime}\right) e^{i m \phi^{\prime}} I_{z}\left(\rho^{\prime}, \phi^{\prime}\right) .
$$

Furthermore, since there is a source inside $V_{\min } \subseteq V$ that generates the same field as $E_{z}$ for $\rho<R^{\text {int }}$, it also follows from the same formulation that

$$
\begin{aligned}
E_{z}(\mathbf{r} & =(\rho, \phi)) \\
& =-\frac{\omega \mu}{4} \sum_{m=-\infty}^{\infty} g_{m} J_{m}(k \rho) e^{-i m \phi}, \quad \rho<R_{\min }^{\mathrm{int}},
\end{aligned}
$$

where $R_{\min }^{\text {int }}$ is the radius of the largest ball centered about the same origin that is disjoint to the minimum source region $V_{\min }$ and is tangential to it in a way that defines a concave boundary of $V_{\min }$.

Let us derive next convex and nonconvex bounds for the minimum source region of a given far field based on the results above. Equations (80) and (81) define the far field while the field for $\rho=a>R$ is defined by (77); that is,

$$
E_{z}(a, \phi)=-\frac{\omega \mu}{4} \sum_{m=-\infty}^{\infty} a_{m} H_{m}^{(1)}(k a) e^{-i m \phi} .
$$

The backpropagation of the far field or the field for $\rho=a>$ $R$ in (87) to a more interior circle of radius $\rho=b<a$ is described by

$$
E_{z}(b, \phi)=-\frac{\omega \mu}{4} \sum_{m=-\infty}^{\infty} a_{m} H_{m}^{(1)}(k b) e^{-i m \phi}
$$

and is meaningful only if the backpropagated field is $L^{2}$ which means

$$
\sum_{m=-\infty}^{\infty}\left|a_{m}\right|^{2}\left|H_{m}^{(1)}(k b)\right|^{2}<\infty
$$

The smallest circle onto which the field can be backpropagated this way defines the minimum circle $B_{\min }$ associated to the given far field $f(\phi)$ or equivalently the multipole 
moments $a_{m}$ and is defined as $B_{\min }=\left\{\mathbf{r} \in \mathbb{R}^{2}:|\mathbf{r}| \leq R_{\min }\right\}$ where

$$
R_{\min }=\inf \left\{b \in \mathbb{R}^{+}: \sum_{m=-\infty}^{\infty}\left|a_{m}\right|^{2}\left|H_{m}^{(1)}(k b)\right|^{2}<\infty\right\} .
$$

Since $H_{m}^{(1)}(z)=\sqrt{2 z / \pi} h_{m-1 / 2}(z)$, it follows from (32) that

$$
H_{m}(z) \sim-\frac{i}{\pi} \Gamma(m)\left(\frac{2}{z}\right)^{m}, \quad m \longrightarrow \infty .
$$

It follows from (90), (91), and a methodology similar to the one leading to main results 1 and 2 that the following $2 \mathrm{D}$ counterpart of main result 2 holds.

Main Result 2' (2D Space). One has

$$
R_{\min }=\sup \left\{b \in \mathbb{R}^{+}: \lim _{m \rightarrow \infty} \Gamma(m)\left(\frac{2}{k b}\right)^{m}\left|a_{m}\right| \neq 0\right\} .
$$

Finally, like in the 3D case, convex bounds for the minimum source region can be estimated as the intersection of the minimum circles corresponding to different origins. In principle one can estimate the minimum convex source region using this approach. We consider next the complementary approach based on an interior diffraction problem that allows the estimation of nonconvex support information up to the (possibly nonconvex) minimum source region.

After a bounding region for the minimum convex source region is estimated by applying the above method (main result $2^{\prime}$ ) for different origins, one can also backpropagate the fields everywhere outside the respective minimum circles of these origins, which accounts for a determination of the radiated field everywhere outside the derived bounding region for the minimum convex source region. The thus computed field corresponds to the unique field due to any source confined to the given bounding region for the minimum convex source region and producing the specified far field. Subsequently one can choose a number of origins all of which are outside the derived bounding region for the minimum convex source region. One can construct circles of finite radius centered at these origins for which the generated field is known in their interior, from the previous backpropagation computations. Nonconvex bounds for the minimum source region can be estimated by considering the backpropapation of the field from any of these circles up to a larger circular region. The largest circular region for which such backpropagation is well-behaved defines a bounding region for the minimum source region which can reveal nonconvex support information. The key idea has been detailed in the previous section for the $3 \mathrm{D}$ case. We outline next only the key main results unique to the $2 \mathrm{D}$ case computations.

Consider for a given origin $O$, chosen outside the derived bounding region for the minimum convex source region, a circular region centered at that origin and residing outside the bounding region for the minimum source region. It follows from (84) and (85) with the substitution of $V$ by the derived bounding region that in the interior of the circular region in question the field obeys an expansion of the form (84). The field at a circle of radius $\rho=b$ inside this region is then

$$
E_{z}(b, \phi)=-\frac{\omega \mu}{4} \sum_{m=-\infty}^{\infty} g_{m} J_{m}(k b) e^{-i m \phi}
$$

Backpropagation to a more exterior circle of radius $\rho=a>b$ is done via

$$
E_{z}(a, \phi)=-\frac{\omega \mu}{4} \sum_{m=-\infty}^{\infty} g_{m} J_{m}(k a) e^{-i m \phi}
$$

which is meaningful so long as

$$
\sum_{m=-\infty}^{\infty}\left|g_{m}\right|^{2}\left|J_{m}(k a)\right|^{2}<\infty
$$

The largest circle of radius $R_{\min }^{\text {int }}$ onto which the field can be backpropagated this way defines a bounding region for the minimum source region associated to the given far field $f(\phi)$ or equivalently the multipole moments $a_{m}$. From (95),

$$
R_{\min }^{\mathrm{int}}=\sup \left\{a \in \mathbb{R}^{+}: \sum_{m=-\infty}^{\infty}\left|g_{m}\right|^{2}\left|J_{m}(k a)\right|^{2}<\infty\right\} .
$$

The minimum source region $V_{\min } \subseteq \bar{B}_{\min }^{\text {int }}$ where $\bar{B}_{\text {min }}^{\text {int }}=\{\mathbf{r} \epsilon$ $\left.\mathbb{R}^{2}: \mathbf{r} \notin B_{\min }^{\text {int }}\right\}$.

Since $J_{m}(z)=\sqrt{2 z / \pi} j_{m-1 / 2}(z)$, it follows from (11) that

$$
J_{m}(z) \sim \frac{(z / 2)^{m}}{\Gamma(m+1)}, \quad m \longrightarrow \infty .
$$

Now it follows from (96), (97), and a methodology similar to the one leading to main results $1-3$ that the following $2 \mathrm{D}$ counterpart of main result 3 holds.

Main Result 3' (2D Space). One has

$$
R_{\min }^{\text {int }}=\inf \left\{a \in \mathbb{R}^{+}: \lim _{m \rightarrow \infty}\left(\frac{k a}{2}\right)^{m} \frac{\left|g_{m}\right|}{\Gamma(m+1)} \neq 0\right\} .
$$

The detailed summary of the algorithm to estimate (possibly nonconvex) bounds for $V_{\min }$ based on the combination of exterior and interior diffraction approaches in $2 \mathrm{D}$ space is similar to the one presented for the $3 \mathrm{D}$ case at the end of the previous section and needs not be (essentially) repeated here. Instead we conclude the paper with a detailed illustrative example applicable to cylindrical scatterers whose length is much larger than the wavelength so that they can be handled via the $2 \mathrm{D}$ Helmholtz equation model.

\section{Example}

We consider electromagnetic wave scattering by a perfect electric conductor (PEC) circular cylinder of radius $R$ centered about the origin. One of the main appeals of the multipole expansion is that it allows treatment of both near and far fields. In this example we consider both near and far probing 
fields combined with sensing in the far zone of the scatterer. It is assumed that the cylinder's length is so much larger than the wavelength that it can be modelled as being infinitely long along the $z$ axis, which facilitates description of the corresponding wave radiation, propagation, and scattering via the 2D Helmholtz equation model.

Consider scattering of the field due to an infinitely long antenna (in the respective $3 \mathrm{D}$ picture) or a point source located outside the PEC circle (in the equivalent 2D framework). The field scattered by the PEC cylinder upon incidence of the field, due to an infinite line of constant current $I_{e}$ located at position $\mathbf{r}^{\prime}$ given in cylindrical coordinates as $\mathbf{r}^{\prime}=$ $\left(\rho^{\prime}, \phi^{\prime}\right)$, is given by $([10$, section 11.5 .5$])$

$$
\begin{aligned}
E_{z} & (\rho, \phi) \\
& =-\frac{k^{2} I_{e}}{4 \omega \epsilon} \sum_{m=-\infty}^{\infty} a_{m} H_{m}^{(1)}(k \rho) e^{-i m \phi}, \quad \rho>R,
\end{aligned}
$$

where

$$
a_{m}=-\frac{J_{m}(k R)}{H_{m}^{(1)}(k R)} H_{m}^{(1)}\left(k \rho^{\prime}\right) e^{i m \phi^{\prime}}
$$

so that

$$
\lim _{m \rightarrow \infty} \Gamma(m)\left(\frac{2}{k b}\right)^{m}\left|a_{m}\right|=\lim _{m \rightarrow \infty} \frac{\Gamma(m)\left(R^{2} / b \rho^{\prime}\right)^{m}}{\Gamma(m+1)}
$$

which means

$$
\begin{array}{ll}
\lim _{m \rightarrow \infty} \Gamma(m)\left(\frac{2}{k b}\right)^{m}\left|a_{m}\right|=0, & b>\frac{R^{2}}{\rho^{\prime}}, \\
\lim _{m \rightarrow \infty} \Gamma(m)\left(\frac{2}{k b}\right)^{m}\left|a_{m}\right|=\infty, & b<\frac{R^{2}}{\rho^{\prime}} .
\end{array}
$$

In view of main result $2^{\prime}$, this means that the smallest ball circumscribing the minimum source region is the ball $B_{\min }=$ $\left\{\mathbf{r} \in \mathbb{R}^{2}: \rho \leq R^{2} / \rho^{\prime}\right\}$. In other words the largest dimension $D$ of the minimum source region must be $D=2 R^{2} / \rho^{\prime}$. Note that, if $\rho^{\prime} \rightarrow \infty$ as is the case for plane wave excitation, we obtain $D=0$ corresponding to a multipole source localized in the origin.

These analytical results assume perfect data, including access to the evanescent spectrum; hence they do not put realistic constraints to avoid unrealistically high near fields and currents (superdirectivity). Furthermore, they hold only for the test origin $(x=0, y=0)$. Still, they establish strict bounds which constitute valuable localization information about the target. In practice the data are essentially finitedimensional. The respective finite-dimensional results are, in fact, more stable and correspond to larger minimum source regions contained inside the original target support. Estimation of target support based on realistic finite dimensional exterior field data and arbitrary test origins is handled numerically by requiring that the functional energy in (89) and (95) exhibits a reasonable value. The concrete approach we adopt in connection with the functional energy (89), that is, main result $2^{\prime}$, is to automatically estimate the peak

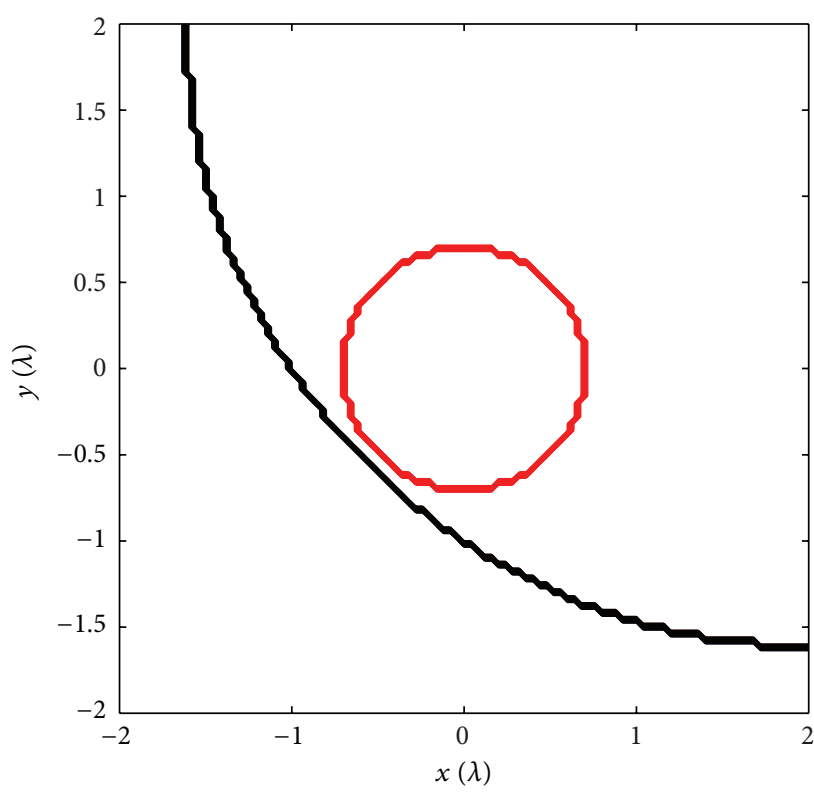

FIGURE 10: Boundary of the minimum source region defined by $R_{\min }$ for test origin $(x=2, y=2)$.

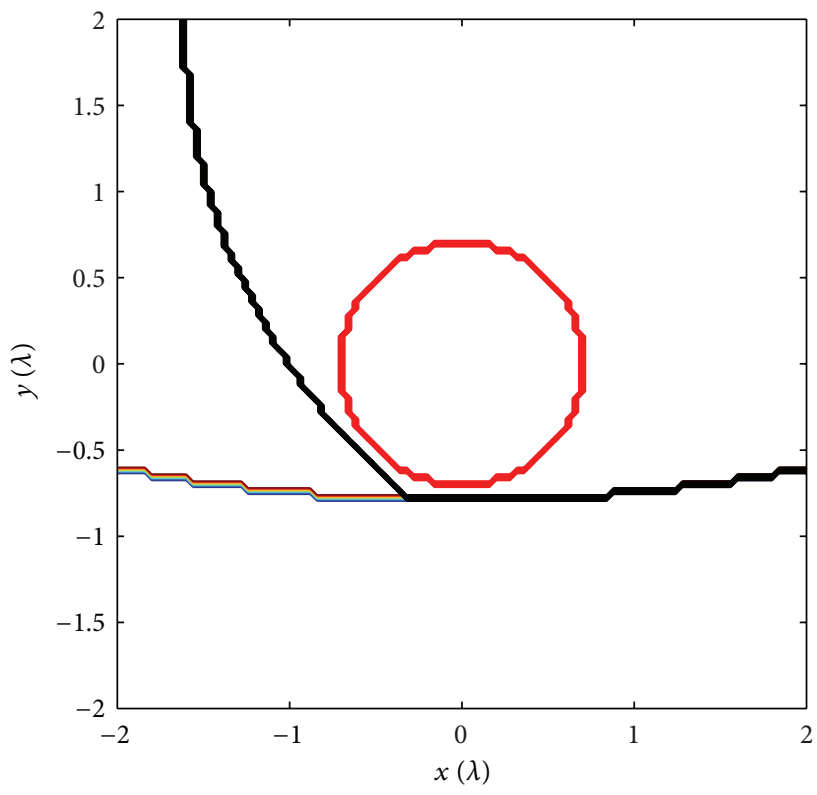

FIGURE 11: Improved boundary of the minimum source region corresponding to two test origins: $(x=2, y=2)$ and $(x=0, y=$ $10)$.

curvature point of the " $\log$ (energy) versus minimum radius $R_{\text {min }}$ " curve and to use this peak curvature point as the calculated minimum radius $R_{\min }$.

For the numerical illustration, we considered a probing field of wavelength $\lambda=1$ due to a source at $(x=-4, y=0)$, and a PEC conductor scatterer of radius $R=0.707$. Figure 10 shows a contour plot of the scatterer and the boundary of the minimum source region obtained for test origin $(x=$ $2, y=2$ ). Figure 11 shows the updated boundary after 


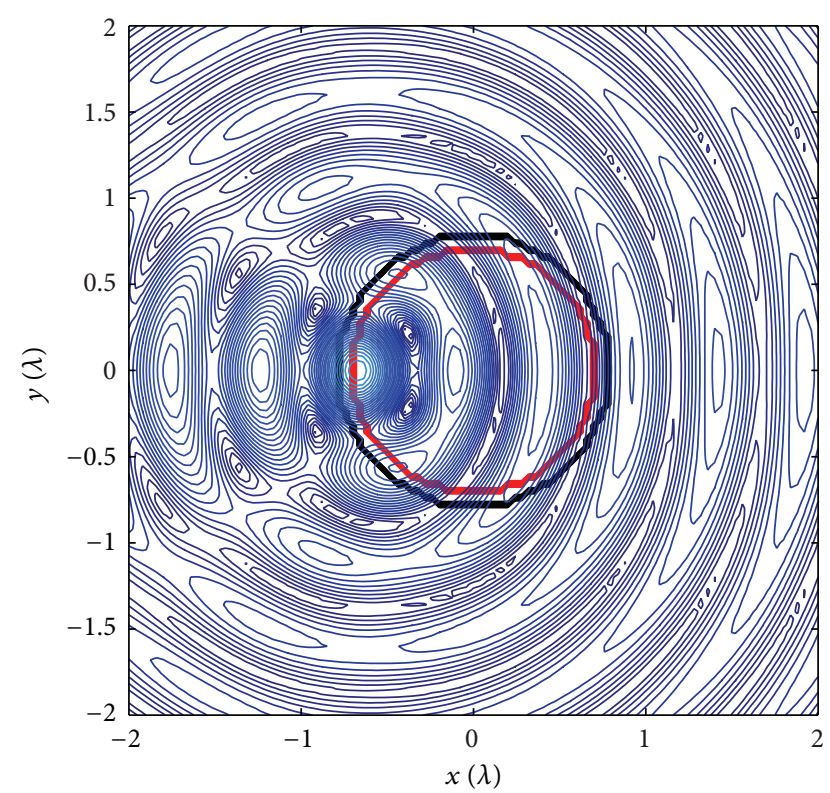

FIGURE 12: Improved boundary of the minimum source region, which essentially coincides with the true target support, corresponding to three test origins: $(x=2, y=2),(x=0, y=10)$, and $(x=0, y=0)$. Also shown is the contour plot of the amplitude of the backpropagated field associated to the field data.

considering test origin $(x=0, y=10)$. Figure 12 shows the computed boundary of the minimum source region for test origin $(x=0, y=0)$, which coincides well with the original scatterer support. In this figure we also show for reference the backpropagated field corresponding to the given field data, which reveals the dominant scattering center at the edge of the true source region. Figure 13 shows the computed boundary obtained from the intersection of the results for the three test origins above plus $(x=-2, y=2)$ and $(x=-10, y=0)$. In this figure we also show for reference the analytically derived boundary for the minimum source region corresponding to this case, which as expected is inside the obtained computational boundary for the minimum region. The shape of the computed minimum region suggests that this approach is robust in estimating the shape in those portions of the scatterer that are within line of sight of the probing source. There appears to be more limited shape information corresponding to the shaded portion of the scatterer. The obtained results suggest that robust estimations of target support are, on the other hand, likely for incident fields carrying a broader spectrum of incident plane waves due, for example, to diversity in the location of the excitation sources or opportunistic multipathing.

\section{Conclusion}

Expressions were derived to estimate the smallest region of localization of a source or scatterer that can produce a given exterior field. The results are based on the multipole expansion. This minimum source region of the given exterior field data can be used to approximately localize sources or scatterers. The derived results permit analytical study

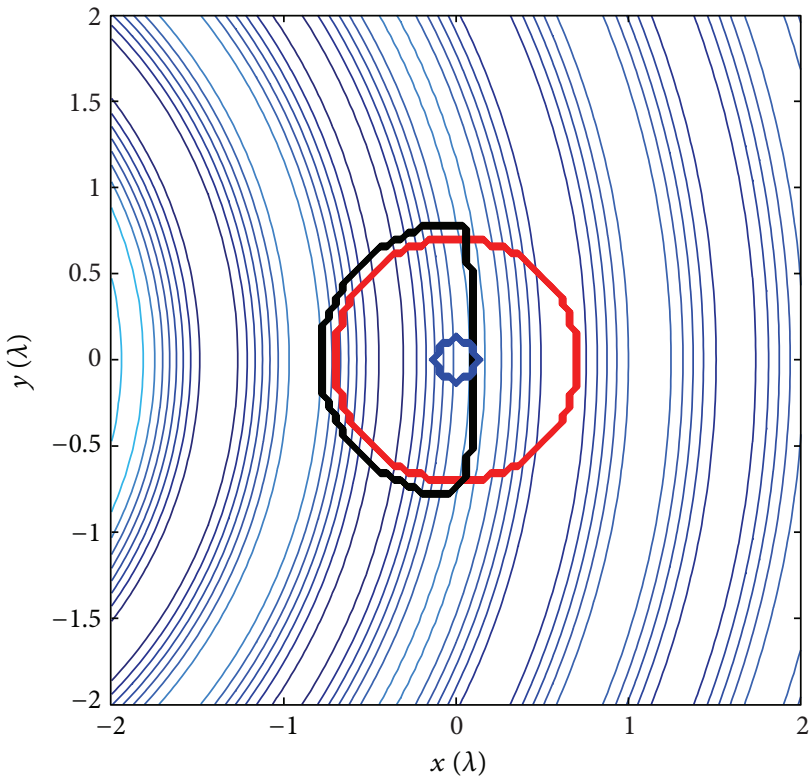

FIGURE 13: Computed boundary of the minimum source region based on test origins $(x=2, y=2),(x=0, y=10),(x=$ $0, y=0),(x=-2, y=2)$, and $(x=-10, y=0)$. Also shown is the contour plot of the real part of the incident field due to the point source at $(x=-4, y=0)$ and the analytical boundary of the minimum source region (small circle in the figure) which ignores the finite-dimensional nature of the field under realistic noise and perturbations.

of imaging and inverse scattering problems that cannot be handled via the plane wave expansion method of previous work. The results were illustrated analytically and numerically for a PEC scatterer excited by line sources in the near or far zone of the scatterer. The numerical results illustrate some of the limitations in shape reconstruction arising from limited views. The same results suggest that target shapes may be robustly estimated when driven by incident fields corresponding to linear superpositions of different sources or incident directions, and this can be done using location diversity and exploiting reverberations in the surrounding medium. We plan to report elsewhere the full vector, electromagnetic generalization of the scalar multipole theory approach to target support estimation that was presented in this paper.

\section{Acknowledgments}

This work was supported by the Air Force Office of Scientific Research under Grant FA9550-12-1-0285. This work was prepared during the author's sabbatical at the Department of Electrical and Computer Engineering at Boston University.

\section{References}

[1] A. D. Yaghjian, T. B. Hansen, and A. J. Devaney, "Minimum source region for a given far-field pattern," IEEE Transactions on Antennas and Propagation, vol. 45, no. 5, pp. 911-912, 1997. 
[2] S. Kusiak and J. Sylvester, "The scattering support," Communications on Pure and Applied Mathematics, vol. 56, no. 11, pp. 15251548, 2003.

[3] C. M. Müller, Foundations of the Mathematical Theory of Electromagnetic Waves, Springer, Berlin, Germany, 1969.

[4] T. B. Hansen and A. D. Yaghjian, Plane Wave Theory of TimeDomain Fields: Near-Field Scanning Applications, IEEE Press, Piscataway, New Jersey, NJ, USA, 1999.

[5] E. A. Marengo, A. J. Devaney, and R. W. Ziolkowski, "Inverse source problem and minimum-energy sources," Journal of the Optical Society of America A, vol. 17, no. 1, pp. 34-45, 2000.

[6] A. J. Devaney and G. C. Sherman, "Nonuniqueness in inverse source and scattering problems," IEEE Transactions on Antennas and Propagation, vol. 30, no. 5, pp. 1034-1037, 1982.

[7] J. D. Jackson, Classical Electrodynamics, John Wiley \& Sons, New York, NY, USA, 3rd edition, 1999.

[8] A. J. Devaney and E. Wolf, "Multipole expansions and plane wave representations of the electromagnetic field," Journal of Mathematical Physics, vol. 15, no. 2, pp. 234-244, 1973.

[9] G. B. Arfken and H. J. Weber, Mathematical Methods for Physicists, Elsevier Academic Press, New York, NY, USA, 6th edition, 2005.

[10] C. A. Balanis, Advanced Engineering Electromagnetics, John Wiley \& Sons, New York, NY, USA, 1989. 

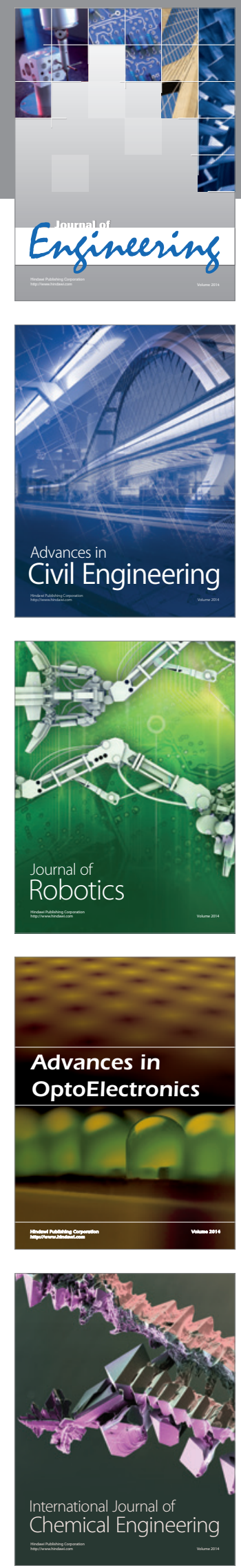

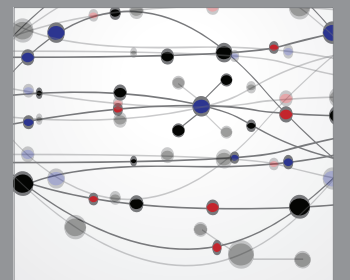

The Scientific World Journal
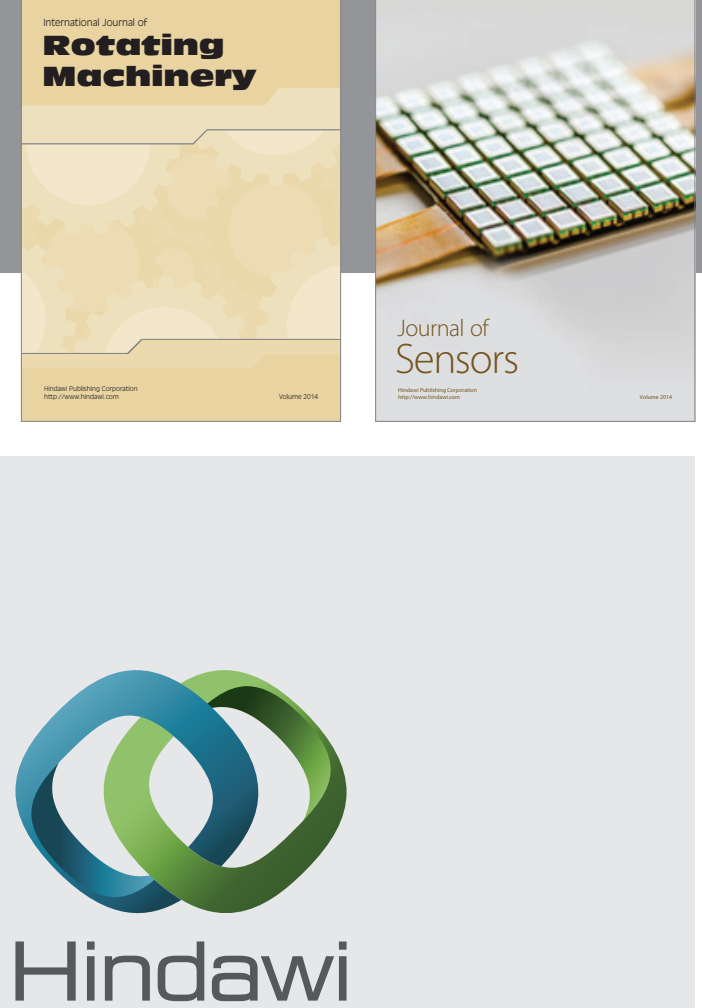

Submit your manuscripts at http://www.hindawi.com
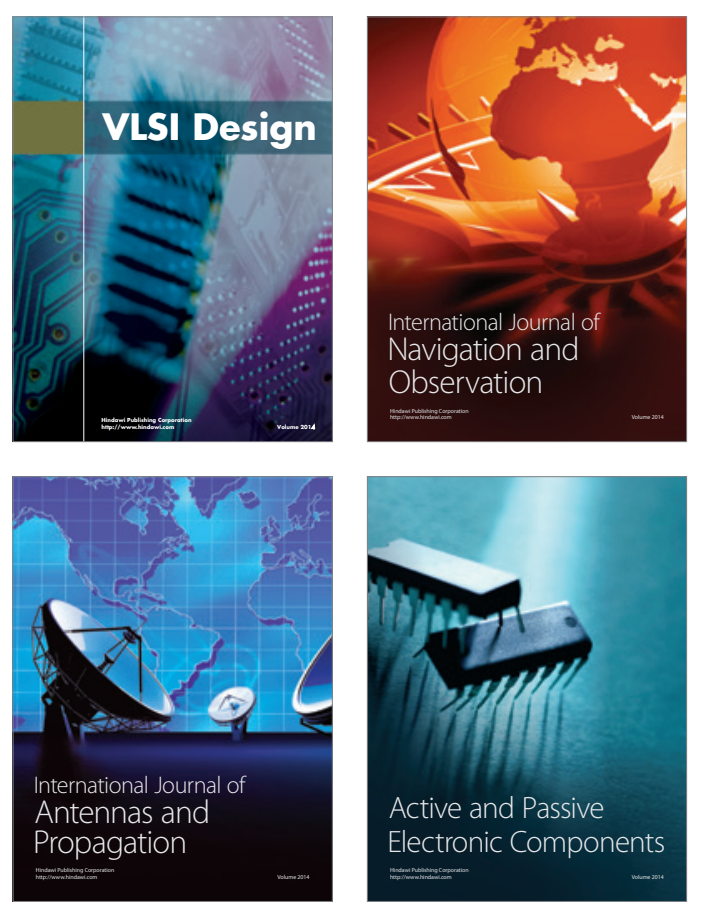
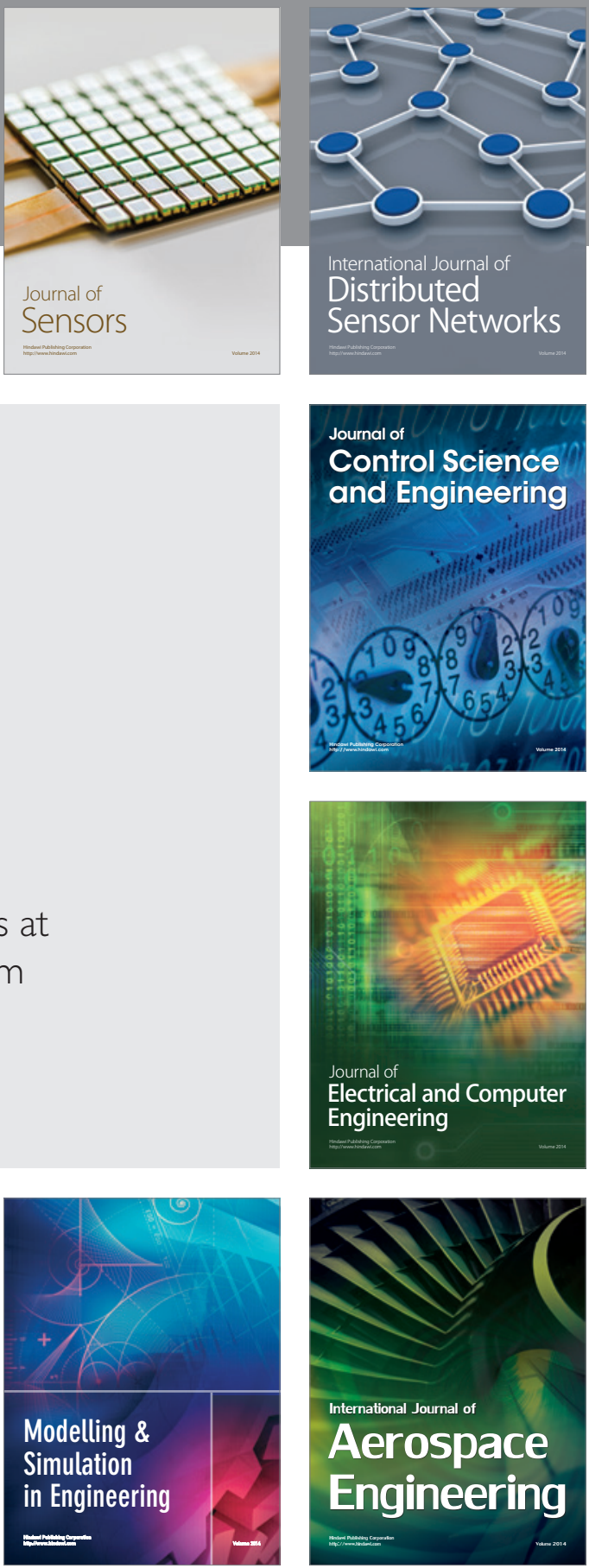

Journal of

Control Science

and Engineering
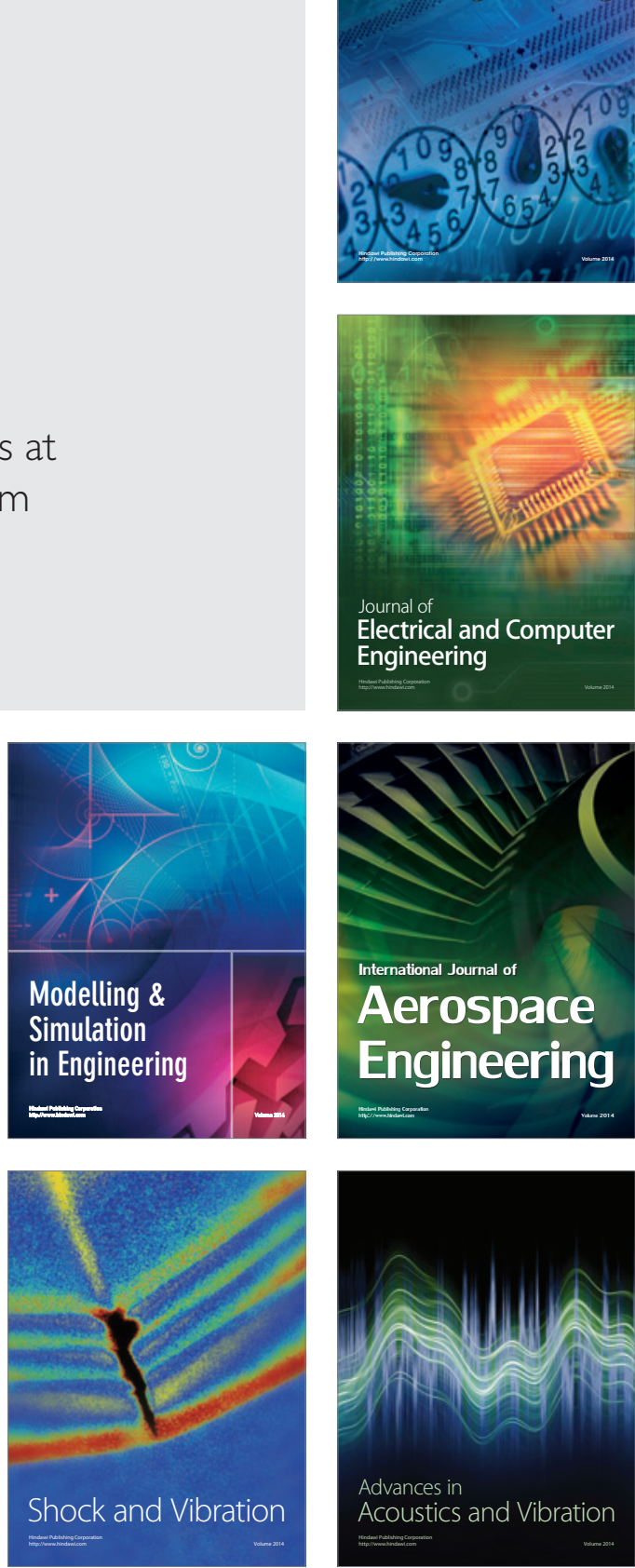